() Ю.Л. Федорченко'

'ФГБОУ ВО Дальневосточный государственный медицинский университет Минздрава России, Хабаровск 2КГБУЗ Городская поликлиника №16, Хабаровск

ОБОСНОВАНИЕ. Лечение инфекции Helicobacter pylori (HP) у больных сахарным диабетом 2 типа (СД2) с хроническими гастродуоденальными заболеваниями (ГДЗ) остается актуальной задачей, учитывая высокую степень инфицированности данных пациентов: от 70 до 90\%.

ЦЕЛЬ. Состояла в изучении эффективности и последствий тройной и оптимизированной последовательной схем антихеликобактерной терапии больных СД2 в сочетании с ГДЗ.

МАТЕРИАЛЫ И МЕТОДЫ. Обследовано 54 больных СД2 и 64 пациента без СД2, последние в качестве группы сравнения, в возрасте от 30 до 60 лет. Проведена гастродуоденоскопия для подтверждения ГД3, хеликобактерную инфекцию определяли уреазным методом и исследованием антигена бактерии в кале. Оценивали динамику клинической картины по балльной шкале GSRS, эндоскопическую ремиссию, эффективность эрадикации НР. Изучали влияние схем антихеликобактерной терапии на синдром избыточного бактериального роста в тонком кишечнике (СИБР) у данных больных. СИБР определяли дыхательным водородным тестом. Сформированы 4 группы обследованных: 1-я группа без СД2 и 2-я - с СД2 получали тройную антихеликобактерную терапию 10 дней; 3-я группа без СД2 и 4-я группа с СД2 - оптимизированную последовательную терапию 14 дней.

РЕзУЛЬТАТЫ. Установлено, что оптимизированная последовательная схема лечения привела к эрадикации НР у больных без СД2 в 90\% случаев, а у пациентов с СД2 - в 85,7\% случаев, что достоверно больше, чем при тройной схеме - 67,6\% и 65,3\% соответственно. Кроме того, клиническая эффективность и эндоскопическая ремиссия также были выше при использовании последовательной схемы лечения. Интерес представляют данные по влиянию эрадикационной терапии на СИБР в тонком кишечнике, который исходно имел место у 44-46\% больных ГД3 без СД2 (в зависимости от группы) и у 69-78\% больных СД2. Только оптимизированная последовательная схема терапии позволила достоверно уменьшить число больных с СИБР в обеих группах.

ЗАКЛЮЧЕНИЕ. Использование в качестве антихеликобактерной терапии больных СД2 с ГДЗ оптимизированной последовательной схемы повышает клиническую эффективность лечения, степень эрадикации возбудителя по сравнению со стандартной тройной терапией.

КЛЮЧЕВЫЕ СЛОВА: хеликобактерная инфекция; сахарный диабет; гастродуоденальные заболевания

\title{
TREATMENT OF HELICOBACTER PYLORI CONTAMINATION IN PATIENTS WITH TYPE 2 DIABETES MELLITUS WITH GASTRODUODENAL DISORDERS
}

(c) Yuri L. Fedorchenko', Marina V. Martynuk²

${ }^{1}$ Far-Eastern state medical university, Khabarovsk, Russia

${ }^{2}$ City out-patient clinic №16, Khabarovsk, Russia

BACKGROUND: The treatment of Helicobacter pylori (HP) infection in patients with diabetes mellitus with chronic gastroduodenal disorders is a substantial problem because of its high incidence.

AIMS: To compare between the effectiveness of triple and optimised consecutive regimens in anti-HP treatment in patients with type 2 diabetes mellitus with gastroduodenal disorders.

MATERIALS AND METHODS: The study included 54 patients with diabetes mellitus and 64 healthy individuals (the control group) aged 30-60 years. Gastroduodenal pathology was confirmed by gastroduodenoscopy; HP contamination was confirming using Marshall's urease method or by finding bacterial antigen in excrements. We studied the dynamics of clinical manifestations using the GSRS scale and showed remission via endoscopy and the effectiveness of HP eradication. We also analysed the effects of anti-HP therapy regimens on the presence of small intestinal bacterial overgrowth syndrome (SIBOS) in patients with diabetes mellitus. SIBOS was diagnosed via the respiratory hydrogen method.

RESULTS: The use of an optimised consecutive regimen resulted in HP eradication in $85.7 \%$ of patients with diabetes mellitus compared with a $65.3 \%$ eradication in patients on the triple therapy. Moreover, clinical improvement and endoscopy-con- 
firmed remission were more frequently observed in patients on the optimised consecutive regimen. A statistically significant decrease in the number of patients with SIBOS was found only in patients who underwent the optimised consecutive therapy regimen.

CONCLUSIONS: This study showed that the optimised consecutive therapy regimen was more effective than the triple therapy in HP eradication.

KEYWORDS: Helicobacterpylory; diabetes mellitus; gastroduodenal diseases

Основным постулатом лечения патологии, ассоциированной с инфекцией Helicobacter pylori (HP), является принцип эрадикации возбудителя. В соответствии с международным консенсусом Маастрихт V (2015), Киотским консенсусом (2015), рекомендациями Российской гастроэнтерологической ассоциации по диагностике и лечению НР у взрослых (2012) регламентированы стандарты антихеликобактерной терапии [1, 2]. Однако, как свидетельствуют многочисленные отечественные и зарубежные исследования, за последнее десятилетие эффективность классических схем первой и второй линии эрадикации упала [3]. Это связано с рядом проблем, главные из которых: рост резистентности НР к метронидазолу и кларитромицину; побочные действия ингибиторов протонной помпы и антибактериальных препаратов [4].

Одной из новых схем эрадикационной терапии является последовательная схема (5 дней ингибитор протонной помпы (ИПП) + амоксициллин, далее 5 дней ИПП + кларитромицин + тинидазол/метронидазол). Эта схема в настоящее время - одна из самых изучаемых схем эрадикации во всем мире, что продиктовано высокими цифрами эффективности такой терапии [5]. В рамках консенсуса Маастрихт V данный протокол эрадикации регламентирован в качестве альтернативы схемы первой линии [2]. Данный консенсус рекомендует также для оптимизации лечения и увеличения эффективности эрадикации пролонгировать лечение схемами первой линии до 14 дней.

Для больных сахарным диабетом 2 типа (СД2) с хроническими гастродуоденальными заболеваниями (ГДЗ) также актуально стоит вопрос об эрадикации НР, т.к. доказана высокая степень инфицирования этих пациентов, от 70 до 90\% [6]. Однако исследования, в которых изучались схемы эрадикации НР у больных СД с ГДЗ, В нашей стране и за рубежом, единичны $[7,8]$. В частности, в доступной литературе нами не найдены работы по использованию последовательной схемы у данных пациентов.

Актуальной является проблема переносимости больными эрадикационной терапии, ее влияния, положительного или отрицательного, на качество жизни пациентов, на состояние микробиоты кишечника. Это особенно важно с учетом модификации схем эрадикации, увеличения длительности лечения и числа антибактериальных препаратов в этих схемах.

\section{ЦЕЛЬ}

Цель исследования состояла в изучении эффективности и последствий тройной и оптимизированной последовательной схем антихеликобактерной терапии больных СД2 в сочетании с ГДЗ.

\section{МЕТОДЫ}

\section{Дизайн исследования}

В открытое сравнительное контролируемое исследование по принципу случайной выборки были включены 118 пациентов, страдающих различными ГДЗ и инфицированные $Н P$, из них у 54 имелся достоверно установленный СД2. 64 пациента с ГД3, но не имевшие СД2, составили группу сравнения. ГДЗ были диагностированы согласно имеющимся стандартам обследования с привлечением специальных методов: эзофагогастродуоденоскопии (ЭГДС), взятия биопсии слизистой желудка и двенадцатиперстной кишки с гистологическим исследованием. Инфицированность НР определяли уреазным тестом (в биоптатах минимум из двух мест - тела и антрума желудка) и методом иммуноферментного анализа (ИФА) с моноклональными антителами для выявления антигенов возбудителя в кале. Причем последний метод был обязателен для контроля за эрадикацией, который осуществляли через 1 мес после окончания лечения.

Все пациенты были разделены на 4 группы: в 1-ю группу вошли больные без СД2, которые получали стандартную тройную схему эрадикации НР. Во 2-ю группу - больные СД2, получавшие также тройную терапию. В 3-ю группу вошли больные без СД2 и в 4-ю - с СД2, которым была назначена оптимизированная последовательная схема эрадикации НР. 1-я и 3-я группы расценивались как контрольные по отношению к больным СД2. В табл. 1 представлена сравнительная характеристика всех 4 групп по результатам рандомизации.

Как видно из табл. 1, пациенты 4 групп достоверно не различались между собой по таким показателям, как возраст, распределение по полу, индексу массы тела (ИМТ), длительности заболевания, распределению по частоте ГДЗ, по методам оценки HP, что делало их максимально однородными за исключением отсутствия или наличия СД2. Единственное различие между группами контроля и группами СД2 было в частоте кардиальной патологии, которая в большей степени встречалась у больных СД2. Но это не могло повлиять на дальнейший ход исследования.

В задачи работы входило определение клинико-эндоскопической и лабораторной эффективности эрадикационных схем. Для оценки клинической эффективности использовали методику оценки гастроэнтерологических жалоб по системе GSRS (Gastrointestinal Simptom Rating Scale), разработанную I.Wiklund [9]. Русскоязычная версия опросника GSRS была создана исследователями Межнационального Центра исследования качества жизни (г. Санкт-Петербург) [10]. Суть методики заключалась в опросе больных по 5 шкалам: DS - диарейный синдром, IS - диспептический, CS - констипационный, AP - абдоминальных болей, RS - рефлюксный. За каждый синдром можно получить максимум от 14 до 28 баллов в зависи- 
Таблица 1. Исходная характеристика больных СД2 и контрольных групп, получавших тройную и последовательную антихеликобактерную терапию

\begin{tabular}{|c|c|c|c|c|c|c|c|c|}
\hline \multirow[b]{2}{*}{ Признак } & \multicolumn{2}{|c|}{ Тройная терапия } & \multicolumn{2}{|c|}{$\begin{array}{c}\text { Последовательная } \\
\text { терапия }\end{array}$} & \multirow[b]{2}{*}{$P_{1-2}$} & \multirow[b]{2}{*}{$P_{3-4}$} & \multirow[b]{2}{*}{$P_{1-3}$} & \multirow[b]{2}{*}{$P_{2-4}$} \\
\hline & $\begin{array}{c}1 \text { группа } \\
\text { контроль, } \\
\text { n=34 }\end{array}$ & $\begin{array}{c}2 \text { группа } \\
\text { СД2, } \\
\text { n=26 }\end{array}$ & $\begin{array}{c}3 \text { группа } \\
\text { контроль, } \\
\text { n=30 }\end{array}$ & $\begin{array}{c}4 \text { группа } \\
\text { СД2, } \\
\text { n=28 }\end{array}$ & & & & \\
\hline Возраст, лет & $52,4 \pm 6,3$ & $54,6 \pm 4,2$ & $47,3 \pm 5,2$ & $52,3 \pm 4,1$ & 0,458 & 0,438 & 0,265 & 0,722 \\
\hline \multicolumn{9}{|l|}{ Пол } \\
\hline мужчины, n (\%) & $14(41,2)$ & $10(38,5)$ & $8(26,7)$ & $11(39,3)$ & 0,376 & 0,321 & 0,244 & 0,558 \\
\hline женщины, n (\%) & $20(58,8)$ & $16(61,5)$ & $22(73,3)$ & $17(60,7)$ & 0,621 & 0,458 & 0,332 & 0,416 \\
\hline ИМТ, кг/M² & $27,5 \pm 3,2$ & $29,5 \pm 2,8$ & $26,4 \pm 2,1$ & $30,2 \pm 3,1$ & 0,578 & 0,345 & 0,389 & 0,421 \\
\hline \multicolumn{9}{|l|}{ Болезни, абс. (\%) } \\
\hline НЭр. гастрит & $11(32,4)$ & $8(30,8)$ & $13(43,3)$ & $9(32,1)$ & 0,434 & 0,465 & 0,433 & 0,211 \\
\hline Эр. гастрит & $16(47,1)$ & $12(46,1)$ & $13(43,3)$ & $13(46,4)$ & 0,786 & 0,278 & 0,465 & 0,390 \\
\hline ЯБ & $7(20,6)$ & $6(23,1)$ & $4(13,4)$ & $6(21,4)$ & 0,146 & 0,277 & 0,132 & 0,178 \\
\hline Ср. длительность болезни, лет & $16,4 \pm 4.2$ & $14,2 \pm 3,6$ & $14,2 \pm 3,6$ & $15,8 \pm 4,2$ & 0,389 & 0,567 & 0,234 & 0,543 \\
\hline \multicolumn{9}{|l|}{ Сопут. болезни, абс. (\%) } \\
\hline ГЭРБ & $14(41,2)$ & $13(59,1)$ & $11(36,7)$ & $10(55,5)$ & 0,089 & 0,078 & 0,099 & 0,121 \\
\hline ПБЗ & $16(47,1)$ & $12(54,5)$ & $11(36,7)$ & $8(44,4)$ & 0,768 & 0,422 & 0,322 & 0,237 \\
\hline ИБС & $8(23,5)$ & $10(45,5)$ & $5(16,7)$ & $12(66,7)$ & 0,034 & 0,021 & 0,235 & 0,267 \\
\hline ГБ & $15(44,1)$ & $20(90,9)$ & $10(33,3)$ & $23(82,1)$ & 0,007 & 0,012 & 0,562 & 0,711 \\
\hline \multicolumn{9}{|l|}{ Исходная оценка НР, абс. (\%) } \\
\hline Уреазный тест & $27(79,4)$ & $18(69,2)$ & $23(76,7)$ & $18(64,3)$ & 0,534 & 0,347 & 0,356 & 0,558 \\
\hline ИФА в кале & $7(20,6)$ & $8(30,8)$ & $7(23,3)$ & $10(35,7)$ & 0,711 & 0,544 & 0,566 & 0,497 \\
\hline
\end{tabular}

Примечания: ГЭРБ - гастроэзофагеальная рефлюксная болезнь, НЭр. - неэрозивный, Эр. - эрозивный, ЯБ - язвенная болезнь, ПБЗ - панкреатобилиарные заболевания, ИБС - ишемическая болезнь сердца, ГБ - гипертоническая болезнь.

мости от частоты симптомов. Для общей оценки жалоб баллы разных шкал суммировались.

Одна из задач нашего исследования состояла в изучении влияния эрадикационных схем на микробиоту тонкого кишечника, на синдром избыточного бактериального роста (СИБР) в тонком кишечнике. Последний встречается довольно часто у больных СД2 [7]. Для выявления СИБР использовали результаты водородного теста с лактулозой, который проводили исходно и через 1 мес после окончания лечения. Водородный тест признан наиболее информативным для оценки наличия СИБР В кишечнике. В качестве прибора для проведения водородного теста применяли «Гастролайзер» (Великобритания), а сама методика не отличалась от стандартной [11].

\section{Критерии соответствия}

Из обследования были исключены лица, страдающие тяжелыми декомпенсированными заболеваниями сердца, легких, печени, поджелудочной железы, кишечника. От всех пациентов было получено письменное согласие на обследование и лечение, согласно приказу Минздрава РФ № 173/1 от 25 июля 2012 г. «Об информированном добровольном согласии на медицинскую помощь».

\section{Условия проведения}

Все пациенты были жителями г. Хабаровска, наблюдались у гастроэнтеролога в ГБУЗ Городская поликлиника №16, у эндокринолога в «Центре эндокринологического здоровья» г. Хабаровска. На базе этих учреждений проводились обследование и лечение больных.

\section{Продолжительность исследования}

Исследование проводилось на протяжении 2 лет. Каждый обследованный перед началом исследованияв течение 2 нед не принимал ингибиторы протонной помпы (ИПП) и минимум 6 мес - антибиотики. После постановки диагноза ГДЗ и выявления инфекции НР пациентам назначалась одна из схем антихеликобактерной терапии согласно дизайну исследования. Для определения эндоскопической эффективности лечения, по его окончанию проводили ЭГДС с оценкой состояния слизистой желудка и двенадцатиперстной кишки. Следует отметить, что больные, помимо эрадикационной терапии, получали антацидную и антисекреторную терапию (только тем ИПП, который был назначен первоначально) до 1 мес. Проведение водородного теста с определением СИБР осуществляли исходно и через 1 мес после окончания антихеликобактерной терапии.

\section{Описание медицинского вмешательства}

Стандартная тройная схема антихеликобактерной терапии включала в себя: омепразол 40 мг/сут + кларитромицин 1 г/сут + амоксициллин 2 г/сут в течение 10 дней. Последовательная схема эрадикации НР была представлена выше, дозировки препаратов такие же, как в тройной схеме + метронидазол - 1 г/сут. Оптимизация данной схемы состояла в увеличении продолжительности лечения: ИПП + амоксициллин 7 дней, далее: ИПП + кларитромицин + метронидазол еще 7 дней. Кроме того, вместо омепразола в данной схеме использовался рабепразол, так как, согласно положению 10 рабочей группы 3 Маа- 
Таблица 2. Результаты лечения тройной антихеликобактерной схемой больных СД2 и контрольной группы

\begin{tabular}{|c|c|c|c|c|}
\hline \multirow[t]{2}{*}{ Признак } & \multicolumn{2}{|c|}{$\begin{array}{c}\text { Контроль (группа 1) } \\
\text { n=34 }\end{array}$} & \multicolumn{2}{|c|}{$\begin{array}{c}\text { Больные СД2 (группа 2) } \\
\text { n=26 }\end{array}$} \\
\hline & исходно & после лечения & исходно & после лечения \\
\hline Больных с НP, абс. (\%) & $34(100)$ & $11(32,4)^{*}$ & $26(100)$ & $9(34,6)^{*}$ \\
\hline Больных с ремиссией по данным ЭГДС, абс. (\%) & - & $24(70,6)$ & - & $16(61,5)$ \\
\hline Общий GSPS, баллы & $56,7 \pm 4,3$ & $38,1 \pm 2,3^{*}$ & $\underline{79,6 \pm 4,1}$ & $54,6 \pm 3,2^{*}$ \\
\hline \multicolumn{5}{|l|}{ Синдромы GSPS, баллы } \\
\hline DS (диарейный) & $14,4 \pm 2,4$ & $10,4 \pm 1,1$ & $\underline{7,9 \pm 1,2}$ & $14,3 \pm 1,1^{*}$ \\
\hline IS (диспепсический) & $17,6 \pm 2,1$ & $10,7 \pm 1,3^{*}$ & $\underline{26,6 \pm 2,1}$ & $11,6 \pm 2,1^{*}$ \\
\hline CS (констипационный) & $8,2 \pm 1,8$ & $14,8 \pm 1,8^{*}$ & $\overline{13,1 \pm 1,3}$ & $12,2 \pm 2,4$ \\
\hline АР (абдоминальных болей) & $6,8 \pm 1,1$ & $3,1 \pm 0,4^{*}$ & $\underline{13,2 \pm 1,6}$ & $4,2 \pm 1,1^{*}$ \\
\hline RS (рефлюксный) & $14,2 \pm 1,3$ & $4,1 \pm 1,1^{*}$ & $\underline{20,8 \pm 1,6}$ & $8,3 \pm 1,4^{*}$ \\
\hline СИБР, абс. (\%) & $15(44,1)$ & $10(29,4)$ & $18(69,2)$ & $13(50)$ \\
\hline
\end{tabular}

Примечания:____ достоверность различий в исходных показателях между группами, p<0,05, * достоверность различий с исходными показателями в каждой группе, $\mathrm{p}<0,05$.

стрихта V, этот ИПП признан наименее подверженным влиянию генотипа СYР2C19 и метаболизирующимся преимущественно в результате неферментативного процесca [2].

\section{Основной исход исследования}

Лабораторной эффективностью эрадикационных схем считались отрицательные результаты ИФА на антигены НР в кале через 1 мес после окончания лечения.

\section{Дополнительные исходы исследования}

Клиническую эффективность лечения оценивали по достоверному снижению показателя GSRS по отношению к исходному через 1 мес от начала терапии ГДЗ. Эндоскопическая эффективность определялась исчезновением эндоскопических признаков заболевания по данным ЭГДС также через 1 мес от начала лечения.

\section{Этическая экспертиза}

На представленное исследование получено заключение экспертной комиссии по вопросам медицинской этики ФГБОУ ВО ДВГМУ (Положение об этическом комитете при научно-плановой комиссии ДВГМУ от 08.01.2014 г., утверждено ректором В.П.Молочным): проведенное научное исследование соответствует общепринятым нормам морали, требованию соблюдения прав, интересов и личного достоинства лиц, принимавших участие в исследовании.

\section{Статистический анализ}

Результаты исследования обработаны с помощью пакета прикладных программ Statistica 10, Excel 2014. Абсолютные величины сравнивали с расчетом средних значений, ошибки средних, с использованием критерия Стьюдента. Для относительных величин применяли точный критерий Фишера. Различия считались статистически значимыми при достигнутом уровне $p<0,05$.

\section{РЕЗУЛЬТАТЫ}

Результаты тройной схемы антихеликобактерной терапии больных СД в сравнении с контрольной 1-й группой представлены в табл. 2.
Следует отметить, что исходно у больных СД2 был достоверно выше общий балл GSRS, чем у пациентов без СД2, а значит, более выраженная клиническая картина ГДЗ. У больных СД2 клинически достоверно чаще встречались диспептический, рефлюксный, синдромы абдоминальных болей, чем в контрольной группе. В свою очередь, диарейный синдром достоверно чаще наблюдался в группе контроля. Запоры наблюдались примерно с одинаковой частотой в обеих группах.

Через 1 мес лечения, в том числе антихеликобактерного, в группе без СД2 достоверно с исходным уменьшились проявления диспептического, рефлюксного синдромов, синдрома абдоминальных болей, но чаще стал наблюдаться констипационный синдром. По синдрому диареи существенных изменений не произошло. В целом общий средний балл GSRS стал достоверно меньше. В группе СД2 на фоне терапии также произошло значимое уменьшение проявлений диспептического, рефлюксного и болевого синдромов, но чаще стала наблюдаться у больных диарея. Общий балл гастроэнтерологических жалоб все же достоверно снизился с 79,6 до $54,6(p<0,05)$.

При проведении ЭГДС после завершения лечения оказалось, что в группе 1 в 70,6\%, а в группе 2 - в 61,5\% случаев имела место положительная динамика в виде заживления язв и эрозий, улучшения гистологической картины со стороны слизистой желудка и двенадцатиперстной кишки. Эффективность эрадикации НР при тройной антихеликобактерной терапии установлена следующая: в группе 1, у больных без СД - 67,6\%, а в группе 2, у пациентов с СД2 - 65,3\% (p>0,05).

Исследование показало, что исходно СИБР достоверно чаще встречался во 2-й группе с СД2 по сравнению с контрольной группой 1. При этом схема тройной терапии привела к уменьшению числа больных с СИБР в обеих группах, но снижение это не было достоверно значимым.

В табл. 3 представлены результаты лечения пациентов с ГДЗ оптимизированной последовательной схемой антихеликобактерной терапии.

Как видно из табл. 3, исходная клиническая картина, по общему среднему баллу GSRS, была достоверно более выражена в группе 4 с СД2, по сравнению с контрольной 
Таблица 3. Результаты лечения оптимизированной последовательной антихеликобактерной схемой больных Сд2 и контрольной группы

\begin{tabular}{|c|c|c|c|c|}
\hline \multirow[t]{2}{*}{ Признак } & \multicolumn{2}{|c|}{$\begin{array}{c}\text { Контроль (группа 3) } \\
\text { n=30 }\end{array}$} & \multicolumn{2}{|c|}{$\begin{array}{c}\text { Больные СД2 (группа 4) } \\
\text { n=28 }\end{array}$} \\
\hline & исходно & после лечения & исходно & после лечения \\
\hline Больных с НР, абс. (\%) & $30(100)$ & $3(10)^{*}$ & $18(100)$ & $4(14,2)^{*}$ \\
\hline Больных с ремиссией по данным ЭГДС, абс. (\%) & & $28(93,3)$ & & $23(82,1)$ \\
\hline Общий GSPS, баллы & $57,2 \pm 3,8$ & $30,2 \pm 2,4^{*}$ & $\underline{76,4 \pm 2,5}$ & $38,6 \pm 2,4^{*}$ \\
\hline Синдромы GSPS, баллы & & & & \\
\hline DS (диарейный) & $13,6 \pm 1,4$ & $4,4 \pm 1,1^{*}$ & $\underline{9,4 \pm 1,2}$ & $3,1 \pm 1,1^{*}$ \\
\hline IS (диспепсический) & $18,2 \pm 1,2$ & $7,8 \pm 1,4^{*}$ & $25,2 \pm 2,6$ & $11,1 \pm 1,3^{*}$ \\
\hline CS (констипационный) & $7,8 \pm 1,3$ & $6,3 \pm 1,2$ & $\overline{10,8 \pm 2,2}$ & $7,9 \pm 2,1$ \\
\hline АР (абдоминальных болей) & $7,2 \pm 1,4$ & $3,1 \pm 0,6^{*}$ & $12,8 \pm 1,3$ & $5,2 \pm 1,3^{*}$ \\
\hline RS (рефлюксный) & $9,8 \pm 1,2$ & $3,6 \pm 1,2^{*}$ & $\overline{18,2 \pm 1,8}$ & $8,4 \pm 1,4^{*}$ \\
\hline СИБР, абс. (\%) & $14(46,7)$ & $6(20)^{*}$ & $\underline{22(78,5)}$ & $6(21,4)^{*}$ \\
\hline
\end{tabular}

Примечания: ___ _остоверность различий в исходных показателях между группами, р<0,05, * достоверность различий с исходными показателями в каждой группе, $p<0,05$.

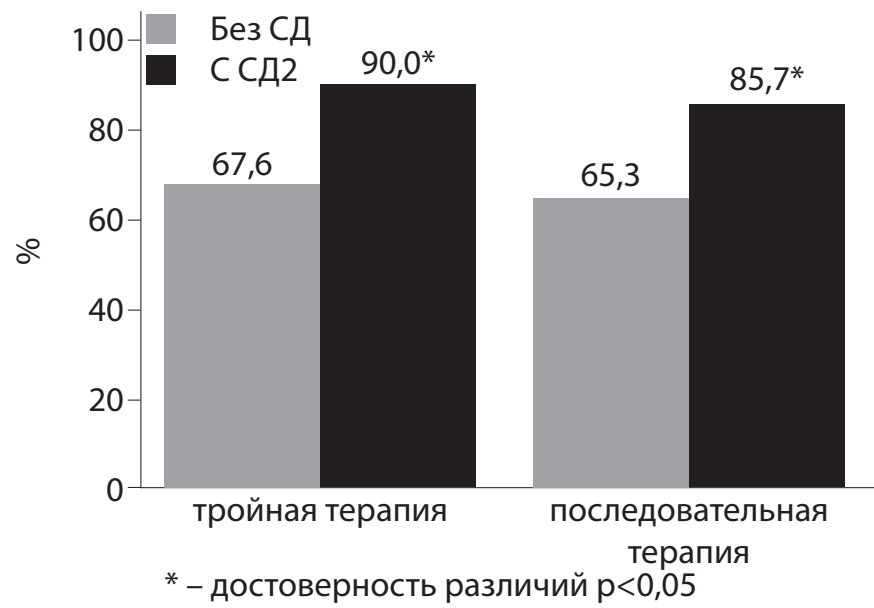

Рис. 1. Сравнение эффективности эрадикации НР при назначении тройной и оптимизированной последовательной схем терапии.

группой 3. У больных СД2 достоверно чаще наблюдались диспептический, болевой, рефлюксный синдромы и реже диарейный. На фоне лечения с использованием последовательной схемы в группе 3 достоверно уменьшились проявления диарейного, диспептического, болевого и рефлюксного синдромов. Такие же положительные изменения произошли в группе 4 больных с СД2, также с достоверной значимостью.

В ходе лечения эндоскопическая ремиссия была достигнута у 93,3\% больных 3-й группы и у 82,1\% пациентов 4-й группы. Эффективная эрадикация H.pylori на фоне оптимизированной последовательной схемы имела место у 90\% пациентов 3-й группы и у 85,7\% больных 4-й группы.

Исходно СИБР в тонком кишечнике, как и в случае с тройной схемой лечения, достоверно чаще наблюдали в группе 4 с СД2 по сравнению с группой контроля 3. Но, в отличие от результатов лечения тройной терапией, при данной схеме как в группе 3, так и в группе 4 имело место достоверное снижение числа больных с СИБР по окончанию терапии.

\section{ОБСУЖДЕНИЕ}

Таким образом, результаты нашего исследования позволили сравнить эффективность тройной и оптими- зированной последовательной схем антихеликобактерной терапии больных с ГДЗ при наличии СД2 и без него. Следует отметить, что, согласно рекомендациям, посвященным проблеме хеликобактериоза, задача лечения заключается в более $80 \%$ эрадикации возбудителя, оптимальным считается от 85 до 90\% [1]. Однако, как свидетельствуют многочисленные отечественные и зарубежные исследования, за последнее десятилетие эффективность классических схем первой и второй линии упала и редко превышает необходимый процент [3]. Как показали наши исследования (рис. 1), в группе тройной терапии как у больных СД2, так и без диабета процент эрадикации НР не достигал и 70\%.

Для больных с СД2 он был ниже, чем в контрольной группе, хотя и недостоверно. В группе пациентов, получающих оптимизированную последовательную терапию, получен больший эффект эрадикации НР: у пациентов без СД2 - 90\%, а с СД2 - 85,7\%. Различия с тройной схемой лечения были достоверны: для больных без СД2 $p=0,035$ и для больных с СД2 - $p=0,044$.

Обе схемы лечения во всех группах привели к уменьшению клинической картины ГДЗ, но в большей степени это наблюдалось в группах оптимизированной последовательной терапии. Для больных СД2 $\triangle \mathrm{GSRS}$ (уменьшение общей суммы баллов) при тройной терапии составил $25,1 \pm 3,2$ балла ( $<<0,05), \Delta \mathrm{GSRS}$ при последовательной те-

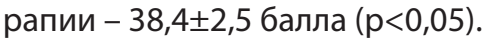

Эндоскопическая ремиссия также была в большем проценте случаев получена во всех группах последовательной терапии по отношению к тройной терапии.

Об эффективности последовательной схемы можно судить по серии клинических исследований, осуществленных в разных странах мира. Так, в Италии и Испании в период с 2003 по 2007 гг. были проведены исследования, включавшие более 100 человек каждое, уровень эрадикации НР достигал при этом 91-95\% [12]. В исследовании Y.S.Kim и соавт. (2011), проведенном в Южной Корее, эффективность 10-дневной последовательной схемы составила 92,6\% по сравнению с 85\% 14-дневной тройной схемой с одинаковыми побочными явлениями [13]. Метаанализ, который включал 36 рандомизированных контролируемых исследований, смог 
продемонстрировать преимущества последовательной схемы, ее более высокую эффективность $(84,1 \%)$ по сравнению с классическим протоколом тройной терапии $(75,1 \%)$ [14]. Но следует отметить, что все перечисленные исследования не касались лиц, страдающих СД2.

По рекомендациям Российской гастроэнтерологической ассоциации (2012), последовательная схема утверждена в качестве альтернативной и высокоэффективной, эффект которой мало зависит от резистентности к кларитромицину. А значит, может применяться в районах, где эта резистентность высока, или не исследована. В этих рекомендациях отмечено, что последовательная схема лечения является многообещающей, и применительно к России требует проведения собственных исследований для уточнения ее места в терапии инфекции НP [1].

Интерес представляют данные по влиянию эрадикационной терапии на СИРБ в тонком кишечнике, который исходно имел место у 44-46\% больных ГДЗ без СД (в зависимости от группы) и у 69-78\% больных СД2. В нашем исследовании только оптимизированная последовательная схема терапии позволила достоверно уменьшить число больных с СИБР в обеих группах.

Исходя из анализа полученных результатов исследования, можно заключить, что использование оптими зированной последовательной схемы лечения больных с хеликобактериозом и ГДЗ имеет существенные преимущества перед классической тройной терапией. Это касается как пациентов без сопутствующего СД, так и с СД2. Для последней группы данные результаты особо значимы, т.к. ранее таких исследований не проводили. В рамках консенсуса Маастрихт V протокол последовательной схемы регламентируется как терапия первой линии, особенно в регионах с высокой резистентностью НР к кларитромицину [2]. Но так как в Хабаровском крае исследований по изучению данной резистентности не проводилось, последовательная схема приобретает особую актуальность.

\section{выводы}

Применение у больных СД2 в сочетании с ГДЗ в качестве антихеликобактерной терапии оптимизированной последовательной схемы позволило повысить эффективность эрадикации НР по сравнению с тройной схемой терапии с 65,3 до 85,7\%.

Использование оптимизированной последовательной схемы эрадикации у больных СД2 с ГД3 имеет преимущества перед тройной схемой по степени уменьшения клинической картины и получения эндоскопической ремиссии у больных.

Назначение оптимизированной последовательной схемы терапии больным СД2 с ГДЗ, в отличие от тройной схемы терапии, позволяет достоверно уменьшить число больных с сопутствующим СИБР в кишечнике.

\section{ДОПОЛНИТЕЛЬНАЯ ИНФОРМАЦИЯ}

Финансирование работы. Финансирование за счет средств бюджета ГБУз Городская поликлиника № 16, г. Хабаровск, частное финансирование Федорченко Ю.Л., Мартынюк М.В.

Конфликт интересов. Авторы декларируют отсутствие явных и потенциальных конфликтов интересов, связанных с публикацией данной статьи.

Участие авторов. Федорченко Ю.Л. концепция, дизайн исследования, анализ данных, написание статьи; Мартынюк М.В. - сбор материала, обработка, анализ данных, написание статьи.

\section{СПИСОК ЛИТЕРАТУРЫ | REFERENCES}

1. Маев И.В., Самсонов А.А., Андреев Д.Н. Инфекция Helicobacter pylori. - М.: ГЭОТАР-Медия, 2016. [Maev IV, Samsonov AA, Andreev DN. Helicobacter pylory infection. Moscow: GEOTAR-Media; 2016. (In Russ.)]

2. Malfertheiner P, Megraud F, O'Morain CA, et al. Management of Helicobacter pylori infection - the Maastricht V/ Florence Consensus Report. Gut. 2017;66(1):6-30. doi: 10.1136/gutjnl-2016-312288

3. Georgopolos SD, Papastergiou V, Karatapanis S. Helicobacter pylori eradication therapies in the era of increasing antibiotic resistance: A paradigm shift to improved efficacy. Gastroenterol Res Pract. 2012;2012:757926. doi: 10.1155/2012/757926

4. Симаненков С.В., Захарова Н.И., Савилова И.В. Сравнительное исследование препаратов Денол и Новобисмол в схемах эрадикации у пациентов с H. pylori-ассоциированными заболеваниями // Экспериментальная и клиническая гастроэнтерология. - 2015. №1. - C. 66-71. [Simonenkov SV, Zaharova NI, Savilova IV. Comparative study of De-nol and Novobismol in regimens of eradication in patients with H.pylori.- associated disorders. Experimental \& clinical gastroenterology. 2015;(1):66-71. (In Russ.)]

5. De Francesco V, lerardi E, Hassan C, Zullo A. Helicobacter pylori therapy: Present and future. World J Gastroin Pharmacol Ther. 2012;3(4):6873. doi: 10.4292/wjgpt.v3.i4.68

6. Мкртумян А.М., Казюлин А.Н., Баирова К.И. Частота и степень инфицированности хеликобактером при сахарном диабете 2 типа // Сахарный диабеm. - T. 13. - №1. - C. 77-79. [Mkrutamyan AM, Kazulin AN, Bairova Kl. Incidence and graveness of helicobacter infection in patients with type 2 diabetes mellitus. Diabetes mellitus. 2010;13(1):77-79. (In Russ.)] doi: 10.14341/2072-0351-6020

7. Осипенко М.Ф., Воронцова Е.С., Жук Е.А. Гастроэнтерологические симптомы при сахарном диабете 2 типа // Экспериментальная и клиническая гастроэнтерология. - 2015. - №3. - С. 84-89. [Osipenko MF, Vorontsova ES, Zhuk EA. Gastroenterological symptom in diabetes mellitus type 2. Experimental \& clinical gastroenterology. 2015;(3):85-89. (In Russ)]

8. Sargýn M, Uygur-Bayramicli O, Sargýn H. Type 2 diabetes mellitus affects eradication rate of Helicobacter pylori. World J Gastroenterol. 2003;9(5):1126-1128. doi: 10.3748/wjg.v9.i5.1126

9. Wiklund I, Bardhan KD, Muller-Lissner S, et al. Quality of life during acute and intermittent of gastroesophageal reflux disease with omeprazol compared with ranitidine. Results from a multicentre clinical trial. The European Study Group. Ital J Gastroenterol Hepatol. 1998;30(1):19-27.

10. Новик А.А., Ионова Т.И. Руководство по исследованию качества жизни в медицине (3-е издание, переработанное и дополненное). / Под ред. Шевченко Ю.Л. - М.: Издательство РАЕН, 2012. [Novik AA, Ionova TI. Guide to the study of quality of life in medicine (3rd edition, revised and supplemented). Shevchenko YuL, editor. Moscow: Publishing House of the Russian Academy of Natural Sciences; 2012. (In Russ.)]

11. Плотникова Е.Ю., Захарова Ю.В. Диагностика и лечение синдрома избыточного бактериального роста // Русский медицинский журнал. - 2015. - Т. 23. - №13. - С. 767-772. [Plotnikova EYu, Zakharova YuV. Diagnosis and treatment of the syndrome of excessive bacterial growth. Russian medical journal. 2015;23(13):767-772. (In Russ.)]

12. Sanchez-Delgado J, García-Iglesias P, Calvet X, et al. Effectiveness of a 10-Day Triple Therapy Combining Potent Acid Inhibition With Amoxicillin and Metronidazole for $\mathrm{H}$. pylori Eradication in Clinical Practice. A Pilot Study. Gastroenterology. 2010;138(5 Suppl 1):S337. doi: 10.1016/S0016-5085(10)61552-1 
13. Kim YS, Kim SJ, Yoon JH, et al. Randomised clinical trial: the efficacy of a 10-day sequential therapy for Helicobacter pylori in Korea. Aliment Pharmacol Ther. 2011;34(9):1098-1105. doi: 10.1111/j.1365-2036.2011.04843.x
14. Feng L, Wen MY, Zhu YJ, et al. Sequential Therapy of Standard Triple Therapy for Helicobacter pylori Infection: An Updated Systematic Review. Am J Ther. 2015;23(3):e880-893. doi: 10.1097/MJT.0000000000000191

\section{ИНФОРМАЦИЯ ОБ АВТОРАХ [AUTHORS INFO]}

Федорченко Юрий Леонидович, д.М.н., профессор [Yuri L. Fedorchenko, MD, PhD, Professor]; адрес: Россия, 680007, Хабаровск, ул. Волочаевская, д 23 [address: 23, Volichaevskaya street, Habarovsk, 680007 Russian Federation]; ORCID: http://orcid.org/0000-0001-7832-0133; eLibrary SPIN: 5703-4303; e-mail: ulfedmed@mail.ru

Мартынюк Марина Владимировна [Marina V. Martynuk, MD]; ORCID: https://orcid.org/0000-0002-9202-6886; eLibrary SPIN: 7875-0158; e-mail: m20675@yandex.com.

\section{ЦИТИРОВАТЬ:}

Федорченко Ю.Л., Мартынюк М.В. Лечение хеликобактериоза у больных сахарным диабетом 2 типа в сочетании с хронической гастродуоденальной патологией // Сахарный диабет. - 2017. - Т. 20. — №6. - С. 427-433. doi: 10.14341/DM8689

\section{TO CITE THIS ARTICLE:}

Fedorchenko YL, Martynuk MV. Treatment of helicobacter pylori contamination in patients with type 2 diabetes mellitus with gastroduodenal disorders. Diabetes mellitus. 2017;20(6):427-433. doi: 10.14341/DM8689 